\title{
Essais
}

Revue interdisciplinaire d'Humanités

Hors-série 1 | 2013

L'estrangement

\section{Comparaison comme estrangement : Machiavel, les anciens, les modernes, les sauvages}

\section{Lucio Biasiori}

\section{(2) OpenEdition}

1 Journals

Édition électronique

URL : http://journals.openedition.org/essais/2001

DOI : 10.4000/essais. 2001

ISSN : 2276-0970

Éditeur

École doctorale Montaigne Humanités

\section{Édition imprimée}

Date de publication : 15 octobre 2013

Pagination : 51-69

ISBN : 978-2-9544269-1-4

ISSN : 2417-4211

\section{Référence électronique}

Lucio Biasiori, «Comparaison comme estrangement : Machiavel, les anciens, les modernes, les sauvages », Essais [En ligne], Hors-série 1 | 2013, mis en ligne le 05 octobre 2020, consulté le 12 octobre 2020. URL : http://journals.openedition.org/essais/2001 ; DOI : https://doi.org/10.4000/ essais.2001 


\section{Comparaison comme estrangement : Machiavel, les anciens, les modernes, les sauvages}

\section{Lucio Biasiori}

1. Tout récemment, Carlo Ginzburg a rappelé l'importance d'un article d'E. H. Gombrich, datant de 1976, qui portait sur la dichotomie entre Renaissance considérée comme époque historique et comme mouvement intellectuel ${ }^{1}$. Tout en montrant les racines historiographiques plutôt qu'historiques de la Renaissance comme époque de redécouverte des valeurs de l'antiquité dans les arts, Gombrich - et Ginzburg avec lui - nous invitait à considérer la catégorie de Renaissance comme un mouvement d'artistes et théoriciens de l'art qui, en réfléchissant sur l'antiquité, se positionnaient de façon polémique vis-à-vis des produits artistiques « barbares ». On peut d'ailleurs utiliser cette dichotomie avec le double de la Renaissance, c'est-à-dire l'Humanisme.

Au siècle dernier deux interprétations majeures s'affrontent : certains auteurs - à l'instar d'Augusto Campana et Paul Oskar Kristeller -, interprètent l'Humanisme en tant que mouvement de " humanistae » - c'est à dire, littéralement, de ceux qui enseignent les " humanae litterae "; d'autres ont insisté plutôt sur la redécouverte de la dignitas hominis après les ténèbres du Moyen Âge, suivant la signification que le mot «Humanisme » avait prise par la suite ${ }^{2}$. Aussi bien dans un cas que dans l'autre, cette période nous apparaît comme dominée par une tension entre un passé conçu comme un modèle et un présent qui prétend à en restaurer la splendeur. De ce fait, ce n'est pas un hasard si c'est à Florence, à la moitié du XV siècle - un chronotope que les deux interprétations acceptent en

1 Carlo Ginzburg, Modernità: una categoria inservibile?, Seminaire organisé par la Fondation Lelio et Lisli Basso (Rome, le 10 décembre 2009). En ligne : http://multimedia.fondazionebasso. it/index.php?option=com_content \&view=article\&id=82:carlo-ginzburg-moderno-unacategoria-inservibile\&catid=108:modernita\&Itemid=150. Ernst H. Gombrich, The Renaissance. Period or Mouvement?, in J. B. Trapp (éd.), Background to the English Renaissance: Introductory Lectures, Londres, Gray-Mills Publishing, 1974, p. 9-30.

2 Augusto Campana, "The Origins of the Word "Humanist" ", in Journal of the Warburg and Courtauld Institute, n 9, 1946, p. 60-73; Eugenio Garin, L'Umanesimo italiano. Filosofia e vita civile nel Rinascimento, Rome-Bari, Laterza, collection « Biblioteca di cultura moderna », 1952. 
tant que moment fondateur - qu'une " querelle des anciens et des modernes " voit la lumière deux siècles auparavant celle, plus célèbre, de l'Académie française ${ }^{3}$. Ce serait une erreur de considérer de telles querelles comme de simples bagatelles académiques : non seulement parce que les partisans de la supériorité des modernes l'étaient également de l'ordre politique, mais aussi parce que l'ouverture imminente du monde européen à de nouveaux espaces géographiques aurait imposé un double tournant à cette confrontation rebattue. D'un côté, la balance aurait vite penché en faveur des modernes; de l'autre, un nouvel acteur était en train de s'imposer : les sauvages.

2. Une place d'honneur dans l'histoire de cette querelle doit être réservée à la Historia de varietate fortunae de Poggio Bracciolini, rédigée entre 1443 et 1449. La supériorité des anciens, d'après Poggio, était moins liée à l'infériorité des exploits des modernes, qu'à l'absence de bons écrivains : "Ce n'est pas qu'on manque d'histoires dignes d'être transmises à la postérité. Ce que nous n'avons pas, c'est le talent des écrivains, qui sachent mettre en relation l'écriture avec la grandeur des événements $»^{4}$. On aurait pu, tout au plus, dénigrer les princes modernes en ce qui concerne la protection des belles-lettres; toutefois, la question de la comparaison entre passé et présent ne concernait pas la grandeur des faits en soi, mais seulement l'adéquation et la correspondance (" aequari et respondere ») entre les faits et leur récit :

Je n'aurais pas le temps de passer en revue tous les exemples de grands hommes, sur lesquels le sort a exercé son pouvoir. Les livres sont pleins d'histoires que l'antiquité a transmises à la postérité. Il y avait en fait beaucoup d'écrivains qui ne permettaient pas que les exploits de leur temps périssent, et chacun les exaltait en les ornant de mots afin qu'on puisse les lire plus volontiers. Et voilà pourquoi les événements du passé nous sont plus connus que tout ce qui se fait de notre époque ; il nous manque les hommes qui mettent en lumière les faits [...]. Pour être sincère, qu'est-ce que nous ont transmis les anciennes annales qui doit être préféré à Tamerlan [...] très puissant général d’Asie, qui, jadis homme privé, à la faveur du sort, fût amené si haut qu'il commandait à une grande partie du monde ?5

3 Le seul travail d'ensemble sur ce thème - malgré ses préjugés nationalistes - Giacinto Margiotta, Le origini italiane de la Querelle des anciens et des modernes, Rome, Éditrice Studium, 1953. Une mise au point in Riccardo Fubini, L'umanesimo italiano e i suoi storici. Origini rinascimentali, critica moderna, Milan, Franco Angeli, 2001, p. 121 et suivantes. Des sommaires indications sur le passage du débat florentin au français in Hans Baron, In Search of Florentine Civic Humanism. Essays on the Transition from Medieval to Modern Thought, Princeton, University Press, 1988, II, p. 96 et suivantes.

4 "Non enim desunt historiae dignae quae posteris tradantur, sed scriptorum facultas, qui rerum magnitudinem scriptis aequent et respondeant rebus gestis " (Poggius Bracciolinii, Opera omnia, réproduction anastatique de l'editio princeps, Turin, Bottega d'Erasmo, 1964, II, p. 34-37).

5 "Dies me deficeret, si omnia recensere vellem illustrium virorum exempla, in quibus fortunae licentia versata est ; referti enim sunt libri ejusmodi historiis, quas memoriae posterorum 
Selon Poggio, donc, la comparaison, loin de se cantonner aux exemples classiques, pouvait être également étendue aux barbares ${ }^{6}$. De ce fait, toute différence qualitative entre les deux cas tombait, non seulement dans le temps mais aussi dans l'espace : ce que Marius avait accompli pouvait être comparé aux exploits des rois de France, mais aussi à ceux de Tamerlan. On pouvait passer en somme de la comparaison entre époques différentes à celle entre peuples différents.

Il ne s'agit pas d'un aspect secondaire du De varietate. L'ouvrage se composait de quatre livres. Au deuxième et au troisième livre, dans lesquels Poggio exposait la toute-puissance de la fortune avec une série d'exemples tirés surtout du pontificat d'Eugène IV, il en ajouta deux : un premier livre, qui était une longue digression sur les ruines de Rome ancienne - où était abordée la question de la transmission des événements historiques - et un quatrième et dernier livre. Celui-ci était consacré au récit du voyage en Inde du marchand vénitien Niccolò de' Conti. Ce dernier, cherchant à se faire pardonner sa conversion à l'Islam lors de ses pérégrinations, avait remis à Poggio, secrétaire du pape, l'exposé de ses péripéties. Dans le dessein de Poggio, cette appendice était justifiée par la volonté de démontrer la toutepuissance de la fortune sur chaque individu, tel l'aventureux Niccolò de' Conti ; de plus, ce dernier livre élargissait le règne du hasard à un monde inconnu et montrait qu'une première forme de comparaison entre les événements relatifs à ce monde et ceux de l'antiquité était possible 7 . Le succès éclatant de cette section de l'ouvrage témoigne de son impact, qui fut remarquable surtout lorsque parvinrent les premières nouvelles des découvertes géographiques à occident. La présence de treize exemplaires du seul quatrième livre dans l'ensemble des trente-huit manuscrits conservés de l'ouvrage, de même que sa circulation autonome sous le titre de India recognita (imprimé à Crémone en 1492), sont autant d'indices du succès immédiat du compte-rendu de Niccolò de' Conti, publié plus tard dans les Navigationi et viaggi de Giovanni Battista Ramusio (1550) et ensuite traduit dans les principales langues européennes ${ }^{8}$.

mandavit antiquitas. Erat scriptorum magna copia, qui suorum temporum res gestas interire non patiebantur, quisque eas extollebat atque ornabat dictis, ut libentius legerentur ; quo factum est ut notiora sint nobis, quae prisca tempora tulerunt, quam quae nostra aetate acta sunt ; desunt enim qui lumen afferant rebus gestis [...] Quid enim antiqui annales attulerunt nobis, vel militum numero, vel robore exercitus, vel disciplina militari, vel rerum gestarum gloria ac varietate praeferendum, si vera fateamur, Tambellano, sic enim vulgus appellat, olim imperatori Asiae potentissimo, quem quondam privatum virum adeo secundae fortunae studium erexit, ut magnae parti orbis imperaret. " (Ibid., p. 37)

6 «Ma un aspetto distingue preliminarmente Poggio rispetto alla storiografia fiorentina di Bruni e a quella italiana di Biondo: l'allargarsi cioè della considerazione alle vicende d'Europa o addirittura esotiche" (Riccardo Fubini, Umanesimo e secolarizzazione da Petrarca a Valla, Rome, Bulzoni, collection "Humanistica », 1990, p. 260).

7 Je ne dirais donc pas qu'il s'agit d'une insertion opérée « de façon plutôt artificielle dans le contexte d'ensemble ", comme l'affirme, en partielle contradiction avec son jugement mentionné dans la note précédente, Riccardo Fubini, (Introduzione, in Poggius Bracciolinii, op. cit., p. 499).

8 En portugais (1502), en espagnol (Séville, 1503, Logrogne, 1529), en anglais (1625) et en hollan- 
3. Comme on l'a remarqué de façon convaincante' , l'influence du passage cité du De varietate sur le préambule au livre II des Discours sur la première Decade de Tite-Live de Machiavel est avérée :

Tous les hommes louent le passé et blâment le présent, et souvent sans raison. [...] La première [raison], c'est qu'on ne connaît jamais la vérité tout entière sur le passé ; [...] la plupart des écrivains se laissent si bien subjuguer par les succès des vainqueurs, que, pour rendre leurs triomphes plus éclatants, non seulement ils exagèrent leurs succès, mais la résistance même des ennemis vaincus ; de telle sorte que les descendants des uns et des autres ne peuvent s'empêcher de s'émerveiller devant de tels hommes, de les louer et de les aimer ${ }^{10}$.

En retravaillant le passage de Poggio, Machiavel y ajouta sa conscience des rapports de force qui président à l'écriture de l'histoire. C'est ça qui l'amena à écrire que les historiens "obéissent à la fortune des vainqueurs ", un jugement qui aujourd'hui a le goût du cliché, mais qui tel n'était pas à l'époque où il venait d'être formulé. Et pourtant, il n'est pas impossible que Machiavel ait su tirer du passage du De Varietate autre chose, quelque chose qui autorisait l'ouverture du dialogue à deux entre les anciens et les modernes à un tiers participant : les sauvages. Telle ouverture demeura toujours liée aux exigences polémiques de Machiavel. D’une part, le mot "barbare " et ses dérivés n'ont jamais de signification positive dans ses œuvres, où ils indiquent tantôt les princes orientaux « dissipateur de toutes les civilisations humaines " (Discours, II, 2), tantôt les « inondations » suivies à la chute de l'empire romain (premier livre des Histoires de Florence), tantôt enfin - en accord avec la propagande politique du pape Jules II - les étrangers qu'il était urgent de chasser afin de rétablir la "liberté d'Italie " (comme on lit dans l'exhortation finale du Prince). D'autre part, lorsque Machiavel compare événements et personnages européens et extra-européens, c'est toujours selon une intention polémique. Il s'agit souvent d'une polémique à caractère religieux : c'est le cas, par exemple, du chapitre XIX du Prince, où Machiavel compare la papauté au sultanat turc (sans se soucier de la contradiction par rapport au jugement ironique sur la singularité de la principauté ecclésiastique que l'on lit dans le chapitre XI) ; ou bien lorsqu'il inclut Moïse dans la célèbre série de bâtisseurs violents de nouveaux états exposée au chapitre VI.

dais (Amsterdam, 1664, Leyde, 1706 et 1707). Pour ces aspects, voir la notice très minutieuse de Francesco Surdich, Dé Conti, Niccolo, in Dizionario biografico degli italiani, Rome, Istituto della Enciclopedia italiana, vol. 28, 1983, p. 457-460.

9 Cette influence a été signalée par Francesco Bausi dans son édition des Discours, Rome, Salerno, 2001, p. 296, n. 11.

10 Discours sur la première Décade de Tite-Live, in Euvres complètes, introduction par J. Giono, texte présenté et annoté par E. Barincou, Paris, Gallimard, 1952, p. 510. 
Il est normal pour nous de penser à la comparaison comme à un procédé neutre, comme à un outil qui sert plutôt à souligner les ressemblances plutôt qu'à accentuer les divisions. L'usage polémique que Machiavel fit de cet outil, nous pousse à questionner les origines sanglantes de la catégorie de comparaison, née au cœur des guerres de religion et des Grandes découvertes ${ }^{11}$.

\section{Francesco Guicciardini (Guichardin) fut sans doute le premier à saisir} pleinement le potentiel comparatif de la méthode de Machiavel. Certes, sa démarche à l'égard de Machiavel est toujours ironique. Du haut de sa fonction de gouverneur de Modène, Guichardin se moque de Machiavel, qui au contraire, de représentant de la République auprès de papes et de monarques se retrouve dans une légation sans importance auprès les franciscains de Carpi. L'échange épistolaire entre ces deux personnages a été depuis longtemps étudié par les chercheurs dans le but de souligner à juste titre leurs différences réciproques et le registre comique dans lequel il s'inscrit. Et pourtant, mis à part ce ton ironique, les mots de Guichardin nous disent bien d'autres choses :

Mon cher Machiavel, quand je pense à votre nouveau titre d'ambassadeur auprès d'une communauté de moines, et que je songe à tous les rois, princes et ducs avec lesquels vous avez négocié, je me rappelle Lysandre qui fut chargé, après tant de victoires et de trophées, de distribuer la viande à ces mêmes soldats qu'il avait si glorieusement commandés, et je me dis que les visages peuvent bien changer, mais que le monde voit toujours les mêmes scènes, et qu'il n'arrive aucun événement qui n'ait eu lieu dans les siècles passés. Mais, parce que les noms et les figures changent sans cesse, il n'y a que les habiles qui les reconnaissent; et voilà le principal mérite de l'histoire, elle vous annonce d'avance et vous met sous les yeux les évènements qui n'ont point eu lieu encore, d'où je conclus, pour raisonner aussi conséquemment qu'un moine, qu'on doit de grands éloges à celui qui vous a chargé d'écrire nos annales, et qu'il faut vous presser de remplir promptement cette commission. Au reste, je crois que votre ambassade ne vous sera pas tout-à-fait inutile; vous aurez employé ce loisir de trois jours à approfondir toute la république des capucins, et vous vous servirez de ce modèle pour le comparer à quelques-unes de vos formes ${ }^{12}$.

11 Carlo Ginzburg, "Provincializing the World. European, Indians, Jews ", Postcolonial Studies, n. $14 / 2,2011$, p. $135-150$.

12 Lettre du 18 mai 1521, in Euvres de Machiavel, traduction nouvelle par T. Guiraudet, seconde édition, Paris, chez Pichard, 1803, t. VI, p. 271-272 (la traduction a été revue par nos soins). "Machiavello carissimo. Quando io leggo e vostri titoli di oratore di republica et di frati e considero con quanti re, duchi e principi voi avete altre volte negociato, mi ricordo di Lysandro, a chi doppo tante victorie e trophei, fu dato la cura di distribuire la carne a quelli medesimi soldati a chi sì gloriosamente aveva comandato; e dico: Vedi che, mutati solum e visi delli huomini et e colori extrinseci, le cose medesime tucte ritornano; né vediamo accidente alcuno che a altri tempi non sia stato veduto. Ma el mutare nomi et figure alle cose fa che soli e prudenti le riconoscono: e però è buona et utile la istoria, perché ti mette innanzi e ti fa riconoscere e rivedere quello che mai non avevi conosciuto né veduto. Di che seguita un sillogismo fratescho: che molto è da comendare chi vi ha dato la cura di scrivere annali; e da esortare voi che con diligenzia esequiate lo officio commesso. A che credo non vi sarà al tutto 
L'ironie qui parcourt cette lettre ne doit pas nous faire oublier qu'elle a été écrite par l'un des premiers lecteurs de Machiavel et l'un des premiers qui a critiqué sa tendance à citer (" allegare ») exemples et textes tirés de circonstances historiques les plus variées. En effet, le lendemain Machiavel, jouant visiblement le jeu de se plaindre du bas état dans lequel il était tombé, ne laissa pas tomber l'opportunité de parler de la comparaison comme base véritable de son discours (" ragionare ») :

Quant à la république des capucins, je ne crois pas que mon voyage me soit tout-à-fait inutile ; $j$ 'ai appris beaucoup de leurs règles et institutions qui ont du bon, je vous assure. Je m'en servirai quelque part, et surtout dans les comparaisons. Lorsque je traiterai du silence, je dirai : ils étaient plus silencieux que des moines au réfectoire. Je pourrai citer ainsi beaucoup d'autres bonnes choses, que je devrai à mon petit séjour parmi eux ${ }^{13}$.

Toutefois, l'emploi de la comparaison entre personnages tirés d'histoires très différents n'a pas seulement un but polémique. Retravaillé per Machiavel, le passage de Poggio deviendra un dispositif permettant de réfléchir sur la diversité des mœurs des populations extra-européennes. Cela se manifeste dans l'usage que de Machiavel fait le marchand et voyageur florentin Filippo Sassetti.

5. La place de Machiavel dans l'outillage mental de Sassetti, qui de l'horizon florentin et toscan du commerce et du Studio pisan s'élança vers le voyage en Inde, n'a jamais fait l'objet d'une étude approfondie ${ }^{14}$. Certes, le rapport qu'il entretient avec Machiavel ne differe pas - dans une première phase au moins de celui que l'on peut constater dans beaucoup d'autres cas de Florentins de la moitié du XVI ${ }^{e}$ siècle ${ }^{15}$. On lit avec avidité Machiavel et on en tire ses propres conclusions; on corrige les propos les plus hardis à l'aide d'un aristotélisme plus

inutile questa legatione, perché in cotesto ocio di tre dì arete succiata tucta la repubblica de' zoccoli et a qualche proposito vi varrete di quel modello, comparandolo o ragguaglandolo a qualcuna di quelle vostre forme. » (Niccolò Machiavelli, Opere, C. Vivanti (éd.), Turin-Paris, Einaudi-Gallimard, 1999, II, p. 377).

13 "Circa alle storie e la repubblica de' zoccoli, io non credo di questa venuta avere perduto nulla, perché io ho inteso molte constitutioni et ordini loro che hanno del buono, in modo che io me ne credo valere a qualche proposito, maxime nelle comparationi, perché dove io abbia a ragionare del silentio, io potrò dire: gli stavano più cheti che i frati quando mangiono; e così si potrà per me addurre molte altre cose in mezzo, che mi ha insegnato questo poco della esperienza " (Niccolò Machiavelli, Opere, op. cit., p. 379).

14 Le nom de Machiavel n'apparaît pas dans l'ouvrage majeur sur ce personnage (cf. Marica Milanesi, Filippo Sassetti, Firenze, La Nuova Italia, collection " Pubblicazioni del Centro di studi del pensiero filosofico del Cinquecento e del Seicento in relazione ai problemi della scienza del CNR, Série 1, Studi ", 1973). Le comparativisme de Sassetti touchait même la langue, de manière qu'il a eté considéré un des pères des études indo-européennes : Cf. Francisco Villar, Los indoeuropeos y los orígines de Europa. Lenguaje y historia, Madrid, Gredos, 1991, passim.

15 C'est le " machiavélisme aristotélique " bien étudié par Giuliano Procacci, Machiavelli nella cultura europea dell'età moderna, Roma-Bari, Laterza, "Collezione storica ", 1995. 
traditionnel, ou bien on les dissimule derrière une profession d'orthodoxie ; plus simplement parfois on occulte la source. Laissons de côté le problème de la présence de Machiavel dans les œuvres historiques et poétiques de Sassetti ${ }^{16}$ et concentrons nous plutôt sur sa correspondance. En commentant l'oraison funèbre de Giambattista Adriani, le fils de Marcello Virgilio, maître et supérieur hiérarchique de Machiavel, Sassetti développa la lecture du De varietate fortunae de Poggio faite par le secrétaire florentin :

Il m’a toujours semblé qu'il est bien difficile de louer un homme privé et policé comme il l'était, et voilà la raison : qu'on nous a étourdis avec les histoires des empereurs, des pontifes et des grands-ducs, car ces histoires, puisque elles sont célèbres, nous parviennent toujours accrues et portées à l'excès. Elles s'accompagnent d'un style grandiloquent et retentissant, [...] ; et lorsqu'on parle de choses ordinaires et dépourvues de tragédie, personne ne s'en satisfait ${ }^{17}$.

16 Il suffit de noter que la Vita di Francesco Ferrucci par Sassetti, le début de laquelle n'est qu'un bref résumé des thèses saillantes contenue dans les Discours et dans le premier livre des Istorie forentine, a été écrite sur le modèle de Vita di Castruccio Castracani : "Niuna sentenza è così vera, come quella è, che per le bocche di ciascuno tutto il giorno risuona, che da uno inconveniente che nasca, molti ne seguono. Fu già lodevole costume appresso a' romani che i cittadini di quella repubblica, liberi dalle fatiche della guerra, esercitassero nella patria i loro propri esercizi; e quelli abbandonando, quantunque volte facesse mestiere ritornassero a guerreggiare [...] Venne meno interamente questo costume allora che Ottaviano Augusto si strinse nelle mani il freno del governo del mondo: imperò che, essendo egli mal sicuro principe assoluto di quel popolo feroce che tanto aveva stimato la libertà, per gettare un saldo fondamento dello imperio suo (conoscendo molte volte desiderare i popoli sommamente quello che è cagione della rovina loro) corroppe con la dolcezza dell'ozio l'animo de' cittadini romani cotanto feroce. E liberandoli dalle fatiche della guerra, tolse loro ogni speranza di mai più rivedere il volto della libertà e privògli di quella gloria che il mondo si aveva fatto suggetto, la quale, partendosi da loro, trapassò a quelle genti che furono elette da lui per la fermezza dello imperio. Da questo successe, in processo di tempo, che l'imperio, dalle mani di coloro che per forza o per inganno se l'erano occupato, trapassò a persone per niuna propia virtù di tanto grado meritevoli, dispensato dal mobile volere degli eserciti barbari, ne' quali era rimasta la potenza dell'armi. Da questo, indi a non molto, derivarono le innondazioni di quelle genti settentrionali, che per tanto tempo infamarono la provincia d'Italia ", cité depuis Filippo Sassetti, Vita di Francesco Ferrucci, V. Bramanti (éd.), Turin, Res, collection "Scrinium. Preziosità letterarie ", 2000, p. 3-4. On peut retrouver une référence à la Mandragola dans une lettre adressée de Pise à son cousin Lorenzo Giacomini le 20 décembre 1570 : cf. Filippo Sassetti, Lettere da vari paesi (1570-1588), V. Bramanti (éd.), Milan, Longanesi, collection "I cento viaggi », 1970, p. 54. Pareil pour sa Difesa di Dante, qui semble rappeler les stylèmes machiavéliens même à ceux qui nient au Dialogo intorno alla nostra lingua la paternité de Machiavel (cf. Mario Martelli, Una giarda fiorentina. Il "Dialogo della lingua" attribuito a Niccolò Machiavelli, Rome, Salerno, collection "I quaderni di filologia e critica », 1978, p. 13-14, n. 6, qui reconnaît la présence du Machiavel de l'Arte della guerra, V, dans le stylème « diversamente e in diversi modi » qui revient in le Dialogo et in la Difesa di Dante par Sassetti).

17 «Io sono stato di parere che sia difficile materia a lodare un uomo privato e molto civile com'egli era, e la cagione è questa: che noi abbiamo stordito gli orecchi alle cose di quegli imperadori e pontefici e granduchi, le quali, con tutto l'essere loro grande, sono sempre aggrandite e recate molte volte allo smoderamento. Accompagnansi queste con lo stile grande e sonoro, tal che ogni cosa strepe e rimbomba, e quando poi si viene a trattare delle cose piane e che non hanno il coturno tragico, ognuno non ne rimane satisfatto », F. Sassetti, Lettere, op. cit.,p. 238. 
On peut également trouver des traces de sa lecture de Machiavel dans le Discorso sul commercio tra $i$ Toscani e $i$ Levantini ${ }^{18}$. La discussion ponctuelle à propos de l'utilité d'établir à Livourne un comptoir avec les Turcs, entraîne ici souvent une réflexion sur la diversité des mœurs. Le schéma machiavélien de lecture de la réalité est également bien visible dans cette partie de l'ouvrage, car la comparaison entre les coutumes des Turcs et des Chrétiens ne semble justifier la diversité de mœurs qu'en raison des différents rapports de forces politiques. Le grand saut de Sassetti du petit monde des académies florentines et de l'université de Pise au vaste théâtre du globe en expansion, est ainsi accompagné d'une réflexion ininterrompue sur Machiavel. Sur le point de partir pour l'Inde, il écrit de Lisbonne à Baccio Valori : "Je ne sais pas si je peux attribuer mon voyage en Inde à la nécessité, à l'inclination, ou à bien à d'autres causes. J'ai trouvé écrit une fois par un homme doué, que la fortune, comme une femme, doit être battue de temps en temps et qu'il ne faut pas se passer d'elle, et que seul de cette manière on peut la maitriser $»^{19}$.

Malgré la volonté d'occulter sa source, - indice possible de la volonté de publier ses lettres - l'écriture de Sassetti laisse en réalité entrevoir le nom de Machiavel, qui, dans le chapitre XXV du Prince, avait en effet comparé la fortune à une femme qu'il faut soumettre ${ }^{20}$. En définitive, parmi les raisons variées qui justifient le départ de Sassetti vers l'Inde (simple curiosité, intérêt marchand, intolérance à l'égard du petit monde de la Florence des Médicis), certaines trouvent sans doute une justification dans les textes de Machiavel.

Dans les études sur Sassetti, on a trop souligné l'opposition entre le milieu florentin et sa culture, telle qui se manifeste après ses voyages ${ }^{21}$. Si l'on garde à

18 L'édition qui fait référence est encore celle du XIX ${ }^{\mathrm{e}}$ siècle, in Lettere edite e inedite di Filippo Sassetti raccolte e annotate da Ettore Marcucci, Florence, Accademia della Crusca, 1855, p. 102-116, sans introduction ni notes, publiée deux ans après la première édition, F. L. Polidori (éd.), in Appendice all'Archivio Storico Italiano, n' 9, 1853, p. 165 et suivantes.

19 « Non so oggi se io mi posso attribuire alla necessità o alla inclinazione, o a che altra causa, la mia tornata in India. Trovai una volta scritto da uomo valente, che la fortuna, come femmina, avea bisogno talvolta d'essere strapazzata e tenersi poco di lei, e così se le metteva il cervello a partito ", Lettere, op. cit., p. 335. Sassetti aurait pu trouver une image similaire de la fortune dans le testament rédigé par son arrière-grand-père, Francesco, qui avait déjà attiré l'intérêt de Aby Warburg dans un essai pionnier paru en 1907, où il soulignait l'" ample échelle des vibrations de l'homme cultivé de la première Renaissance ", prêt à prendre les formes hybrides du centaure et à choisir comme devise l'exhortation à la fortune pour qu'elle soit clémente (" mitia fata mihi ") aussi que l'orgueilleuse prétention d'avoir cette déesse bien en main ("à mon pouvoir », récitait son ex-libris). Aby Warburg, Les dernières volontés de Francesco Sassetti, in Essais florentins, avec une présentation d'Eveline Pinto, Paris, Klincksieck, 1990, p. 167 et suivantes.

20 J'ai proposé une nouvelle interpretation de ce celèbre passage machiavélien dans ma thèse encore inédite (Letture di Niccolò. Storia e fortuna di Machiavelli, Thèse de "Perfezionamento" soutenue sous la direction de M. Carlo Ginzburg le 10 octobre 2011, Pise, École Normale Supérieure).

21 L'un des ses meilleurs biographes affirme que " $\mathrm{i}$ libri di un tempo sembrano nel loro insieme accantonati a tutto vantaggio dei testi storico-scientifici » (Vanni Bramanti, «Filippo Sassetti e il viaggio della scrittura ", op. cit., p. 351). 
l'esprit la dichotomie présente dans le début du Prince, on peut affirmer que pour Sassetti l'« expérience des choses modernes » l'emporte sur la " continuelle leçon des anciennes ». En réalité, cette distinction ne fut pas vraiment opératoire chez Machiavel et moins encore chez un lecteur comme Sassettii ${ }^{22}$, qui le 6 février 1586 écrivait de Kochi, dans le sud-ouest de l'Inde, à Alessandro Rinuccini d'avoir trouvé (chez un vendeur d'allumettes) et ensuite lu le Courtisan ${ }^{23}$. Même au milieu de l'Inde donc, Sassetti n'arrêtait pas ses lectures italiennes, au contraire il lisait (ou mieux, il disait de lire) ce qu'il n'avait pas lu et ses lectures agissaient dans le regard qu'il portait sur le monde asiatique.

En rapportant les coutumes militaires des populations locales, Sassetti se rappela encore une fois de Machiavel. Voici ce qu'il écrivait, toujours de Kochi, à Pietro Spina :

Ce sont tous des gens de guerre, et lorsque leur chef ou roi meurt en bataille ils sont obligés d'aller mourir selon la volonté du seigneur. Ceux qui sont destinés à la mort sont appelés amocchi, et le roi qui en possède de plus est le plus puissant car, étant contraint à la guerre, il envoie à la mort contre les ennemies une troupe de ces gens-là, laquelle, ne voulant pas mourir sans vengeance et devant mourir de toute façon, est terriblement intrépide. Ne diffère pas de ce comportement, au moins dans son intention, le sacrifice qui fit de soi-même l'un des consuls romains dans la guerre contre les Latins, alors que son aile quittait déjà le champ de bataille ${ }^{24}$.

Le personnage évoqué dans les dernières lignes est Publius Decius, qui s'était sacrifié lors de la bataille qui eut lieu en 341 av. J.-C à côté du Vésuve. L'épisode est reporté parTite-Live (VIII, 9), mais il est plus probable que Sassetti se rappelât du passage des Discours (II, 16) où Machiavel relate ce même événement :

La victoire la plus décisive que les Romains aient jamais remportée dans aucune guerre sur aucun peuple, est celle qu'ils obtinrent contre les Latins, sous le consulat de Torquatus et de Décius. Ceux-ci, pour avoir perdu cette bataille, devinrent esclaves ; par conséquent les Romains le seraient devenus, s'ils

22 Une division trop nette opère aussi, à mon avis, Marica Milanesi, Filippo Sassetti, op. cit., p. 59-60: « la partenza per la penisola iberica segna lo spartiacque anche nella vita culturale del Sassetti. [...] I testi letterari, per lungo tempo predominanti passano in secondo piano ».

23 Filippo Sassetti, Lettere, op. cit., p. 510 : «Del Cortegiano, del quale scrisse quel conte (abbatteimi a trovarlo qua sur una bottega d'uno che vende zolfanelli); e non avendo mai letto quell'opera, la nuova cortigiania dell'Aspro mi vi fece calare per vedere come e' si rifaceva di questa giornea ". L'Âpre était le nom de plume de Francesco Bonciani à l'Académie des Alterati. Le passage fait référence à la tâche de gentilhomme de cour obtenue par ce dernier auprès du cardinal Gondi.

24 "Sono tutti gente di guerra e quando il loro capitano o re muore in battaglia, sono obrigati andare a morire a volontà del lor signore: e chiamansi questi tali già destinati alla morte amocchi, e quel re che più ne tiene è più possente perché, stretto nella guerra, manda a morire contro ai nemici una banda di questa gente, qual pare a lui, i quali, non volendo morire senza vendetta, e avendo a morire a tutti i partiti, fanno impeto terribile. Non fu dissimile a questo modo di fare, o almeno all'intenzione, un sacrificio che di se stesso fece uno de'consoli romani nella guerra de' latini, ritirandosi già il suo corno della battaglia ", Lettre à Pietro Spina, Kochi, janvier 1584, in Filippo Sassetti, Lettere, op. cit., p. 409. 
n'avaient pas été vainqueurs. [...] On remarque encore, dans le courant de cette journée, deux évènements jusque-là sans exemple, et qui depuis ne se virent presque plus. Pour affermir le courage des soldats, les rendre plus dociles au commandement et plus déterminés dans l'action, des deux consuls l'un se tue lui-même, l'autre fait mourir son fils. La ressemblance que Tite-Live trouve dans les deux armées, consistait à avoir combattu longtemps ensemble, et avoir même langue, même discipline, mêmes armes, même ordre de bataille, identité de nom pour les divisions et pour leurs chefs. Il fallait donc, tout étant égal d'ailleurs pour le courage et pour les forces, qu'il survînt quelque chose d'extraordinaire qui affermît et rendît plus opiniâtre la constance des uns que des autres; car c'est à cet opiniâtreté que l'on doit les victoires, comme nous l'avons dit ailleurs ${ }^{25}$.

Ce qu'il faut souligner dans les récits de cet épisode, ce n'est pas seulement l'insistance dans les deux cas sur le rôle de la nécessité. C'est notamment le contexte dans lequel l'épisode fait son apparition dans les Discours qui marqua la mémoire de Sassetti. En effet, le dispositif de la comparaison était explicite dans le titre de Discours II, 16 - "Combien les armes modernes diffèrent (si disformino) des anciennes ${ }^{26}$ - rendant possible ainsi la lecture de l'exemplum historique dans cette perspective. Comme nous le verrons, le succès de Machiavel et son entrée dans le Panthéon des auteurs et des textes que les européens ont utilisé pour comprendre - et soumettre - les peuples extra-européens, ne sont pas sans relation avec l'auteur lui-même et ses textes. On peut affirmer - contrairement à l'opinion d'une critique orientée essentiellement vers le lecteur et le processus d'appropriation - que ce succès était lié à la présence dans ces textes d'une disposition à comparer les peuples, les événements, les institutions qui relevaient de temps et d'espaces les plus variés.

6. Cet aspect - jusqu’à présent ignorée ${ }^{27}$ - de la fortune littéraire de Machiavel ne concernait pas seulement sa réception italienne. Lorsqu'il retraçait les étapes de son voyage chez les Indiens Tupi du Brésil, le calviniste Jean de Léry compara la cruauté des sauvages avec celle de ses compatriotes :

25 Discours, op. cit., II 16, p. 552-553. "La più importante giornata che fu mai fatta in alcuna guerra con alcuna nazione dal Popolo romano, fu questa che ei fece con i popoli latini nel consolato di Torquato e di Decio. Perché ogni ragione vuole che, così come i latini per averla perduta diventarono servi, così sarebbero stati servi i romani quando non l'avessino vinta. (...) Vedesi ancora come nel maneggio di questa giornata nacquono due accidenti non prima nati e che dipoi hanno radi esempli: che di due consoli, per tenere fermi gli animi de' soldati ed ubbidienti a' comandamenti loro e diliberati al combattere, l'uno ammazzò sé stesso, e l'altro il figliuolo. La parità che Tito Livio dice essere in questi eserciti era che, per avere militato gran tempo insieme, erano pari di lingua, d'ordine e d'armi: perché nello ordinare la zuffa tenevano uno modo medesimo, e gli ordini e i capi degli ordini avevano i medesimi nomi. Era dunque necessario, sendo di pari forze e di pari virtù, che nascesse qualche cosa istraordinaria che fermasse e facesse più ostinati gli animi dell'uno che dell'altro; nella quale ostinazione consiste, come altre volte si è detto, la vittoria " (Opere, op. cit., I, p. 363-364).

26 "Quanto i soldati de' nostri tempi si disformino dagli antichi ordini».

27 Même dans les exhaustifs livres de Giuliano Procacci, Machiavelli nella cultura, op. cit. et de Sydney Anglo, Machiavelli-The First Century. Studies in Enthusiasm, Hostility, Irrelevance, Oxford, University Press, collection « Oxford-Warburg Studies », 2005. 
Leurs haines sont tellement invétérées qu'ils demeurent perpétuellement irréconciliables. Surquoy on peut dire que Machiavel et ses disciples (desquels la France à son grand malheur est maintenant remplie) sont vrais imitateurs des cruautés barbaresques : car puisque, contre la doctrine Chrestienne, ces Athéistes enseignent et pratiquent aussi, que les nouveaux services ne doivent jamais faire oublier les vieilles injures : c'est à dire, que les hommes tenant du naturel du diable, ne doivent point pardonner les uns aux autres, ne monstrent-ils pas bien que leurs cœurs sont plus félons et malins que ceux des tigres mêmes ${ }^{28}$.

Entre le voyage et le compte-rendu, vingt ans s'écoulèrent. À l'enthousiasme du voyageur succéda le pessimisme du calviniste ${ }^{29}$ : les sauvages devinrent en quelque sorte les maîtres de celui qui avait été accusé d'avoir fomenté avec ses écrits immoraux et athées les affrontements religieux et politiques de cette époque. Frank Lestringant a remarqué que "le démarrage foudroyant de son livre, Léry le doit peut-être pour une part à la publicité donnée par son collègue, le pasteur Urbain Chauveton, qui lui fit écho dès 1579 dans la traduction française de l'Histoire nouvelle du Nouveau Monde de Girolamo Benzoni ${ }^{30}$. Il est difficile de surestimer l'importance de la traduction de Benzoni par Chauveton. Toujours Lestringant a souligné le rôle décisif de ce texte dans l'invention du «bon sauvage " américain et la création d'une légende noire antiespagnole. Et pourtant, personne n'a jamais souligné suffisamment le rôle fondamental de l'image de Machiavel dans l'élaboration de ces deux stéréotypes négatif : dans la préface de Chauveton à la version latine du livre de Benzoni, adressée à Théodore de Bèze, le successeur de Calvin, le secrétaire florentin devînt le maître des Conquistadores, après avoir été peint par Léry comme l'élève des atrocités des Indiens :

En asservissant ces peuples et en le régissant après les avoir asservis, ils se montrèrent disciples de Machiavel plus que du Christ [...] et croyerais-tu qu'ils aient commencé par la ruse de Machiavel, ou bien que celui-ci ait pris ses théorèmes de leurs méfaits en Italie. Puisque Machiavel, dans ses commen-

28 Jean de Léry, Histoire d'un voyage fait en la terre du Brésil (1578), édition, présentation et notes par J.-C. Morisot, index des notions ethnologiques par L. Necker, Genève, Droz, collection "Les classiques de la pensée politique », 1975, p. 196. Deux cents ans après, un autre français, le théologien Antoine-Joseph Pernety, renversa entièrement la comparaison établie par Léry entre les sauvages des Amériques et les disciples de Machiavel : non seulement les premiers n'avaient rien à enseigner aux secondes, mais ils auraient à apprendre par ceux-ci les moyens de défense contre la férocité des Conquistadors ["Malhereux Mexiquains, malhereux Péruviens, pourquoi n’aviez-vous pas été à l'école de nos Machiavels! ", Antoine-Joseph Pernety, Examen des Recherches philosophiques sur l'Amérique et les Américains, Paris, G. J. Decker, 1771, I, p. 237-239, cité in Antonello Gerbi, La disputa del Nuovo Mondo. Storia di una polemica (1750-1900), Milan-Naples, Ricciardi, 1955, p. 109]. Sur le comparativisme de Pernety est à voir Furio Jesi, Mitologie intorno allilluminismo, Milan, Edizioni di comunità, collection "Saggi di cultura contemporanea ", 1972, passim.

29 C'est la version donnée par Frank Lestringant, Le Huguenot et le sauvage. L'Amérique et la controverse coloniale, en France, au temps des guerres de religion (1555-1589), Genève, Droz, 2004³, p. 77 et suivantes.

30 Ibidem, p. 162 
taires où il a dressé un tyran plutôt qu'un prince, le convainc entre autres choses de ne pas adorer honnêtement Dieu, tout en prenant néanmoins une allure de piété ${ }^{31}$.

Suivait une statistique relative aux cas où les Espagnols avaient mis en pratique les abominables maximes machiavéliennes ${ }^{32}$. Il faudrait poursuivre les enquêtes sur la naissance de cette légende noire antiespagnole à partir de la légende noire machiavélienne, notamment sur la manière dont ces deux modèles négatifs s'entrecroisent. Même si on se limite au cas de Chauveton, il est très probable de déceler dans la référence négative à Machiavel l'influence du bréviaire de l'antimachiavélisme français, l'Anti-Machiavel (1572) d'Innocent Gentillet, qui avait réuni les préceptes les plus dangereux du Florentin dans une série d'aphorismes tirées du Prince et des Discours ${ }^{33}$. Selon Lestringant, Chauveton laissa tomber toute référence à Machiavel dans le passage de la version latine à celle française de sa traduction, parue un an plus tard et dédiée à Henri III. Mais est-ce bien vrai qu' " en s'élevant en 1579 jusqu'à l'oreille du roi, Chauveton supprimait toute allusion à l'Anti-Machiavel de son coreligionnaire dans une préface réécrite de bout en bout ${ }^{34}$ ? En relisant cette préface, il est assez aisé, au contraire, de repérer des références directes au secrétaire florentin :

31 «Ita enim et subigendis illis populis et, postqum subegerant, regendis [...] Machiavelli discipulos verius quam Christi se esse proderent [...] istos ex Machiavelli ludo profectos, vel istum ex horum praeclaris in Italia facinoribus Theoremata sua hausisse credideris. Quum enim in commentariis illis quibus Tyrannus verius quam Principem instituit Machiavellus, suadeat inter alia, ut quum ex animo Deum non colat, pietatis tamen externam speciem prae se ferens. " (Praefatio, in Novae Novi Orbis Historiae, apud Eustatium Vignon MDLXXVIII, pages non numérotées).

32 "Monet Machiavellus non posse melius provinciam aut agrum recens captum sub imperio teneri, quam si deductis in eum coloniis veteres incolae pellantur [...] id quoque strenue ab Hispanis praestitum [...] Idem Tyrannicus doctor praecipit, Nulla ratione melius civitatem aut provinciam posse et subigi quam si malis moribus impleatur : [...] At ne istud quidem Machiavellus melius scriptis, quam Hispani factis expressere, vulgaria haec, insuetos vino barbaros ebrietate mollire et vincere [...] Praeter haec Machiavellus docet nulla ratione facilius in pace ac fide contineri et prohiberi a rebellione subditos quam si inopia et labore cohibeantur. Mirum quam avide arreptum hoc dociles isti ad vitia discipuli in rem atque usum verterint. ", Praefatio, op. cit. " Machiavel prévient qu'il n'y a meilleure façon de garder une province ou un territoire assujetti récemment que d'y implanter des colonies, après avoir chassé les anciens habitants [...] et les Espagnols ont fait précisément cela [...]. Toujours ce docteur en l'art de tyrannie, enseigne qu'on ne peut dans aucun cas dompter une ville ou une provence sans la combler de mauvais mœurs. [...] Et les Espagnols l'ont accompli dans les faits mieux que Machiavel l'a exposé dans ses écrits, de soûler ces barbares pas accoutumés au vin et les avoir ainsi vaincus. De plus, Machiavel enseigne qu'on ne peut pas maintenir en paix et en fidélité les sujets, ni les impêcher de se révolter, plus aisement qu'en les contraignant à la liberté et au efforts. C'est incroyable l'empressement avec lequel ces disciples si dépravés ont transferé cet enseignement dans la pratique et dans les coutumes".

33 Particulièrement à la $3^{\mathrm{e}}, 5^{\mathrm{e}}$ et $32^{\mathrm{e}}$ proposition de la troisième partie.

34 Frank Lestringant, Le Huguenot et le sauvage, op. cit., p. 179. 
Sire, ie ne say point de doute que plusieurs ne treuvent estrange, qu'une personne de si basse qualité, \& de si petite suffisance que moy, ait osé vous presenter si peu de chose que cecy. Et encore avec cela ie crain bien, que de tant de bons nez qu'il y ha en France, les uns ne me blasment de quelque presumptueuse temerité : \& que les autres memsems ne se rient [...]. Je confesse que tout cecy est peu de chose, voire que ce n'est rien, \& mesmes que tout ce qui peut partir d'un si petit esprit que le mien, est trop bas pour atteindre à la grandeur d'un Roy, mesme d'un tel Roy que vous. Mais aussi faut-il confesser, que tout ce qui est haut en ce monde, ne mesprise pas tousiours ce qui est bas. Le Soleil, quelque haut monté \& magnifique qu'il soit, si ne desdainge-il pase d'espandre ses rayons ça bas, $\&$ de contempler les plaines ${ }^{35}$.

Le lecteur accoutumé aux textes de Machiavel n'hésitera pas à reconnaître dans ces lignes une réécriture assez fidèle de la lettre dédicatoire du Prince à Laurent de Médicis $^{36}$. Entre la dédicace au chef calviniste et celle au fils de Catherine de Médicis, la référence à Machiavel perdait la spécificité donnée par le regard huguenot de Gentillet et changeait de signe : de la condamnation on passait à la citation allusive. Il est bien possible que cet emploi du « vrai » Machiavel - celui qui établissait dans les Discours la comparaison entre la religion virile des Romains et celle ascétique et lâche des Chrétiens - ait contribué à marquer la différence entre l'Histoire nouvelle et l'Historia nova. L'attention de la première aux " grandes conformitez et similitudes entre les mours et coutumes des Indiens orientaux et des Occidentaux, autant en la police comme en la Religion ", allait en effet bien au-delà de la polémique anti-machiavélienne et antiespagnole ${ }^{37}$.

35 Histoire nouvelle du nouveau monde, par Eustace Vignon MDLXXIX, ff. ii $\mathrm{r}$ - iv r.

36 Je transcris ici les parties de la dédicace du Principe les plus proches au texte de l'Histoire nouvelle: «Et ne faut point que l'on m'impute à presomption, si un homme de basse condition e qualité entreprend de discourir sur le reglement du fait des Princes : car, tout ainsi que ceux qui iugent de l'assiette d'un pays, descendent volontiers és plaines et bas lieux pour considerer la nature des montagnes et places eslevees, et pour contempler celles des bas endroits montent sur le haut des montagnes: Semblabement pour bien cognoistre le naturel des peuples, il faut estre Prince, et pour entendre celuy des Princes, il est necessaire d'estre du rang du peuple (...) Et si du sommet de sa hauteur quelquefois elle tourne sa face vers ces beaux (sic) lieux, elle cognoistra clairement combien, sans mon merite, ie souffre de longue main un grand et continuel maltraitement de fortune " (Le prince de Nicholas Machiavel, traduction par Jacques Gohory, Paris, 1571, p. 2-3). « Né voglio sia imputata prosunzione se uno uomo di basso et infimo stato ardisce discorrere e regolare e' governi de' principi; perché cosí come coloro che disegnano e' paesi si pongono bassi nel piano a considerare la natura de' monti e de' luoghi alti e, per considerare quella de' luoghi bassi, si pongano alto sopra' monti, similmente, a conoscere bene la natura de' populi, bisogna essere principe, e, a conoscere bene quella de' principi, conviene essere populare. [...] E se vostra Magnificenzia da lo apice della sua altezza qualche volta volgerà li occhi in questi luoghi bassi, conoscerà quanto io indegnamente sopporti una grande e continua malignità di fortuna " (Opere, op. cit., I, p. 118). Ces passages n'étaient pas présents dans le texte original de Benzoni, même si la dédicace à Scipione Simonetta commençait avec une possible réminiscence machiavélienne : "Sogliono il più delle volte, Illustrissimo Signor mio, i saggi scrittori [...] » (cité in Girolamo Benzoni, La historia del mondo nuovo, rééd. anast. de l'édition vénitienne de 1572, Graz, 1972, f. ii r.). Le passage correspondait au début du Principe : "Sogliono, el più delle volte, coloro che desiderano acquistare grazia appresso uno Principe [...]» (Opere, op. cit., I, p. 117).

37 Histoire nouvelle, op. cit., ff. ii $\mathrm{r}$. 
7. Chez les calvinistes français, l'emploi de Machiavel ne se limitait pas à la condamnation de l'impérialisme espagnol ni à la mise en œuvre d'outils de lecture des rapports entre "police » et « religion » dans le Nouveau Monde. On sait que le refus de l'idée de mission civilisatrice avait été une des raisons du retard de la France par rapport à l'Espagne dans la colonisation des Amériques. Dieu ayant prévu que certains peuples étaient hors du plan du salut, les civiliser équivalait à contredire ce plan ${ }^{38}$. Ce combat pour la légitimité de la colonisation fût également marqué par la réflexion de Machiavel. Dans ses Ragguagli di Parnaso (1613-1615), Traiano Boccalini avait construit l'allégorie de la France en utilisant des arguments contraires à l'expansion coloniale, sans doute tirés des écrits de Machiavel ${ }^{39}$. Cependant, on ne pouvait pas oublier le chapitre III du Prince, où Machiavel avait exhorté le prince des monarchies mixtes à " envoyer des colonies " pour maintenir " une province non conforme par rapport à la langue, aux coutumes et aux ordres "; Chauveton, dans la Historia nova, avait rappelé avec effroi ce chapitre en le citant parmi les différentes leçons données par Machiavel aux Espagnols. Dans quelle mesure, donc, ce qu'on a appelé "le tournant de 1580 " $^{40}$, c'est-à-dire le passage du refus de la colonisation à l'engagement colonial en fonction anticatholique et anti-Habsbourg, personnifié par des lecteurs de Machiavel comme DuplessisMornay et La Popelinière, fût aussi, en partie, le résultat d'une mise à jour des propos du chapitre III du Prince? Si, comme l'affirme Lestringant, l'avis de Duplessis-Mornay vis-à-vis de la politique coloniale de la France, changea grâce à son amitié avec Philip Sidney, il n'est pas impossible que ce dernier - lecteur acharné de Machiavel $^{41}$-, ait suggéré à son ami - à la lumière du Prince - la nécessité d'" envoyer des colonies ".

Le lien entre engagement colonial - surtout dans ses implications économiques et commerciales - et réflexion sur Machiavel semble plus explicite dans le cas de La Popelinière. Corrado Vivanti avait examiné le développement de

38 Giuliano Gliozzi, Adamo e il Nuovo Mondo. La nascita dell'antropologia come ideologia coloniale: dalle genealogie bibliche alle teorie razziali (1500-1700), Florence, La Nuova Italia, collection "Pubblicazioni del Centro di studi del pensiero filosofico del Cinquecento e del Seicento in relazione ai problemi della scienza del CNR, Serie 1, Studi », 1977.

39 Sur ce thème, il est à voir Luciano Stecca, Il Mondo Nuovo rovina del Vecchio? Traiano Boccalini, Louis du May e le Indie Occidentali, in E. Balmas (éd.), La scoperta dell'America e le lettere francesi, Milan, Cisalpino, collection "Quaderni di Acme ", 1992, p. 165-179, qui ne fait aucune référence à Machiavel. Tout le Ragguaglio LXII de la Centuria terza (La Monarchia di Francia, esortata a popolar le Indie, risponde ch'ella in casa sua già coltiva con la zappa e con l'aratro le sue miniere d'oro) découle du chapitre XIX du Livre II des Discours (Che gli acquisti nelle republiche non bene ordinate, e che secondo la romana virtù non procedano, sono a ruina, non ad esaltazione di esse).

40 Frank Lestringant, Le Huguenot et le sauvage, op. cit., p. 193 et suivantes.

41 Irving Ribner, Machiavelli and Sir Philip Sidney, Chapel Hill, University of North Carolina, unpublished $\mathrm{PhD}$ dissertation, 1949. 
l'idée de « civilisation " chez La Popelinière ${ }^{42}$. Toutefois, cette étude ne prend pas en compte un texte manuscrit, tout dernièrement publié, Le ContreMachiavel (postérieur à 1587) ${ }^{43}$. De sa lecture émerge un lien très étroit entre l'anti-machiavélisme et la réflexion sur le Nouveau Monde : tout en constatant la proximité dangereuse entre la vision négative de la nature humaine affichée par Machiavel et celle d'un certain nombre de théologiens de son temps (une référence explicite à ses coreligionnaires calvinistes), La Popelinière distinguait entre « la Nature première et seconde altérée de son premier estat » :

Aucuns sont d'avis qu'on peut doner icy regle certaine sur ces deux avis soit ou non que le Monde ait esté fait c'est à dire qu'on se peut représenter la première communication des hommes purs et simples avec les corrompus exemples de plusieurs peuples par nous descouvers au nouveau Monde dit l'Amérique et terres septentrionales. Car nos gens les ont treuvé tant simples, humains, aimables et en général non seulement esloquens mais si contraires aux detestables passions de ceux qui les descouvrirent que s'ils eussent eu la vraye conoissance de Dieu ils les eussent jugé issus des Anges et vrays enfans de Dieu. Mais comm'un tableau net et neuf recoit aisemant les premiers couleurs qu'on luy peust doner : ils ont esté si bien aprins et tellemant faconés soit d'Instruction exemples et autorités soit par force et tyrannie indigne de nostre Religion qu'ils commencent à ne faire deshoneur à nos puans Chretiens ${ }^{44}$.

Par le truchement de la critique à Machiavel La Popelinière semble ainsi avoir changé d'avis en ce qui concerne le projet de colonisation, dont il avait parlé quelques années auparavant dans les Trois Mondes (1582). Les "puans Chretiens » ont en fait gâché l'état de " telles bonnes Gens » desquels « les Grecs et les Romains et autres semblent avoir prins le modèle de leurs Isles fortunées qu'ils ont tant célébré par leurs escrits pour en avoir ouy parler neantmoins plus que pour les avoir connus et pratiqué de si près que nous ». Ce n'était pas seulement la nature non corrompue de ces peuples qui justifiait la réfutation des " lâches » doctrines de Machiavel sur la méchanceté de la nature humaine. Les Grandes découvertes avaient montré les erreurs du secrétaire florentin, même en ce qui concerne sa vision de l'immutabilité du monde : "Et pour de ces Peuples nouveaux tirer conséquence à tous les anciens de ce vieil Monde il ne faut estimer que le Monde aie tous-jours esté tel qu'il est ny la face de la terre semblable à celle qui se montre à nous ».

La polémique contre le préambule du livre I des Discours sur l'immutabilité du monde est évidente. Mais le Machiavel que La Popelinière refuse était un Machiavel lu à travers le filtre déformant de Gentillet. Pour preuve on peut

42 Corrado Vivanti, "Alle origini dell'idea di civilità. Le scoperte geografiche e gli scritti di Henri de la Popelinière ", in Rivista storica italiana, ${ }^{\circ} 74,1962$, p. 225-249.

43 Henry L. Voisin de la Popelinière, $D u$ Contre Machiavel au Contre-Prince de Machiavel, édition établie et annotée par B. Lourde, Genève, Droz, collection « Les classiques de le pensée politique ", 2010.

44 Ibid., p. 69. 
citer le fait que la Popélinière pour soutenir sa thèse des mutations ininterrompues du monde fait usage des arguments tirés du chapitre des Discours (II, 5) consacré à l'éternité du monde :

Ainsi que toutes choses ont tous-jours changé changent et changeront tousjours à l'avenir tant par accidens particuliers que la suite du temps amène par alterations du naturel que la Mort suit, laquelle neantmoins leur fait changer de forme plus que de naturel et principale qualité tesmoin la Vicissitude qui se voit en toutes les choses de l'Univers, que Generaux soit par Inondations et déluges marins et pluviaux plus ou moins grans les uns que les autres soit par Incendies et Contagions d'un ou plusieurs pais, soit par Trembleterre qui abisme tantost une ville tantost un et plusieurs pais soit par autres inconveniens espouventables. Lesquels estans par plusieurs fois et de tous temps avenus à tous les peuples de ce Monde differemmant toutesfois et à diverses fois ne faut doubter que tous n'aient une ou plusieurs fois en si longue suite de siècles esté changé ${ }^{45}$.

Comme Machiavel l'avait enseigné donc, le monde avait changé maintes fois mais il demeurait toujours le même. La Popelinière le savait, et sa connaissance de Machiavel était en réalité plus approfondie que celle qu'il pouvait tirer de l'œuvre de Gentillet, auquel il faisait pourtant allusion dans le titre de son ouvrage. Comme pour Chauveton, il y avait pour La Popelinière deux Machiavel : celui déformé de Gentillet et un autre, qui lui était parvenu à travers un circuit plus large et plus souterrain et qui accompagnait ses lecteurs vers des chemins inattendus.

8. Dans la France de la seconde moitié du XVI e siècle, Machiavel était en effet une figure double. Pas seulement le "Tyrannicus doctor ", mais aussi l'auteur qui pouvait offrir des clés de lecture pour comprendre la pluralité du monde en expansion. Probablement cette réception de Machiavel a été orientée par la première traduction française des Discours (limitée au premier livre) publiée par Jacques Gohory en 1544, quelques années avant donc que la France devienne le berceau de l'anti-machiavélisme. Dans sa dédicace, Gohory soulignait explicitement les potentialités cognitives de la méthode comparative de Machiavel :

Il vous rapporte premierement en peu de parolles la singularité de l'histoire Romaine selon que Tite-Live l'a descripte, puis sur icelle il debat les profondes matieres vivement d'une part et d'autre, pour en fin se resoudre en quelque hault paradoxe politique, et en ce faisant vous descouvre, entierement les secretz de ce grand gouvernement, lequel a conquis et assubjecty le monde. Mais quand le propos s'y adonne, il parle des Egyptiens, des Grecz, des Turcz, des François, Allemans, Espanolz, Angloys et surtout des seigneuries d'Italie, declare les perfections et imperfections de tous ces Royaumes et republiques de renon. Tellement que ces devis sont un vray miroir de l'histoire universelle .

45 Le premier Livre des Discours de l'Estat de Paix et de guerre, Paris, Denys Janot, 1544, a 4 v. 
Une telle volonté de comparer les formes politiques de peuples divers devait surtout attirer l'attention du Gohory ethnographe. Celui-ci aurait en effet traduit l'année suivante (sur la base de la version italienne parue à Venise en 1534) l'un des plus célèbres récits de voyage du début du XVI siècle, attribué à Gonzalo Fernandez Oviedo ${ }^{46}$. L'analogie entre Machiavel et le Nouveau Monde n'allait pas seulement dans la direction d'un usage des œuvres du Florentin en tant que " miroir de l'histoire universelle ». Il pouvait arriver aussi que le texte même des Discours puisse être lu à travers le filtre de l'expérience des découvertes. Le travail de Enea Balmes sur Gohory traducteur de Machiavel néglige le " discours pour ainsi dire téchnique [...] l'usage du texte bladiano ou giuntino, la liberté et fidélité du traducteur, évaluation des éventuelles additions, commentaire ou arrangements introduits dans la traduction française $»^{47}$. Ces aspects ont été au contraire analysés, avec sa sagacité habituelle, par Sydney Anglo qui, pourtant, juge trop sévèrement le Gohory traducteur ${ }^{48}$. Mettre en lumière les nombreuses inexactitudes de l'adaptation (c'est le cas de l'atténuation de l'impitoyable jugement de Machiavel sur l'église catholique) ne doit pas conduire à sous-estimer certains choix de traduction délibérés. Par exemple, lorsque les " montagnards encore peu civilisés " des Discours I, 11 deviennent les " gens sauvages, demourans es boys, desertz ou montaignes, qui ne sçavent ne mal ne bien ${ }^{49}$, il s'agit probablement moins d'un malentendu que d'un court-circuit entre le texte des Discours et la réflexion sur le Nouveau Monde.

Gohory saisissait un élément bien présent dans les textes machiavéliens, qui allait vite sombrer, sans plus refaire surface. Derrière cette perméabilité entre découvertes géographiques et œuvres de Machiavel il n'y avait pas seulement la grande curiosité de Gohory, comme le voudrait la critique orientée vers le processus d'appropriation du lecteur. Son interprétation - on vient de le voir - était guidée par des textes - le Prince et les Discours - qui étaient structurés par la comparaison. Pourtant, la liberté Gohory lecteur de Machiavel, était limitée non seulement par le texte, mais aussi par le contexte, en particulier par le rapport qu'il entretenait avec ses patrons. Aimant se présenter comme " le solitaire ", Gohory passa au contraire sa vie à la recherche de réseaux personnels qui pouvaient lui assurer les moyens de subsistance et surtout une renommée à la hauteur de ses qualités. Au début des années 1570 , il prêtait service chez la puissante famille des Affaitati, marchands et banquiers originaires de Crémone, qui pratiquait le commerce du sucre entre Lisbonne et Madère. Tout comme les peintres et sculpteurs de son temps,

46 L'Histoire de la terre neuve du Peru en l'Inde Occidentale, Paris, P. Gaultier, 1545.

47 Enea Balmas, Jacques Gohory traduttore del Machiavelli (con documenti inediti), in Studi Machiavelliani, Vérone, 1972, maintenant in Id., Saggi e studi sul rinascimento francese, Padoue, Liviana, 1982, p. 23-73, cité à la page 67.

48 Sydney Anglo, Machiavelli, op. cit., p. 216-225.

49 Le premier Livre des Discours, op. cit., f. 30v. 
il était partagé entre patronage et tradition iconographique. Son portrait de Machiavel en ethnographe relevait donc aussi bien de l'image donnée par Machiavel lui-même dans ses œuvres que du goût de ses patrons.

9. La triangulation anciens/modernes/sauvages a fait l'objet d'un ouvrage récent de François Hartog, dont je suis tributaire ${ }^{50}$. Toutefois, le Machiavel d'Hartog n'est pas la figure dont je viens de dessiner les traits; il reste renfermé dans le dialogue à deux entre anciens et modernes. Plus précisément, ce Machiavel émerge comme un solitaire, puisque " en définissant l'histoire comme comparaison entre passé et présent, Machiavel opère un mouvement de va-et-vient entre la Rome antique et la Florence contemporaine, chacune sert à construire l'intelligibilité de l'autre ». On peut sans doute admettre que les Grandes découvertes n'ont pas laissé de trace dans les œuvres du Florentin ${ }^{51}$; toutefois, cela n'a pas empêché à Machiavel d'être présent dans l'esprit de ceux qui assujettirent ce monde, (comme La Popelinière), ou de ceux qui, comme Sassetti, pensèrent la diversité des cultures avec les outils forgés par la lecture de ses ouvrages de ses ouvrages. Machiavel portait sur la réalité un regard qui était encore celui d'un homme du Vieux Monde ; et pourtant, les cas d'étude ici examinés nous montrent qu'il était possible d'employer ce regard dans le but d'interpréter et de maîtriser une réalité différente, celle mise en lumière par les découvertes. L'utilisation du filtre machiavélien dans la réalité amérindienne est sans aucun doute un exemple de l'adaptation des schèmes culturels européens au Nouveau Monde ${ }^{52}$. Cependant, même la réflexion sur Machiavel en sortit transformée, et je suis persuadé que le futur historien du machiavélisme devra tenir compte d'un Machiavel qui franchit l'Atlantique avec les Conquistadores, les missionnaires et les simples voyageurs ${ }^{53}$.

Je voudrais conclure cette intervention avec les mots d'un autre possible protagoniste de cette journée d'études, qui ne me semblent pas faire fausse note ici. Je pense à Marcel Proust, qui écrivait dans À l'hombre des jeunes filles en fleurs :

50 François Hartog, Anciens, modernes, sauvages, Paris, Gallimard, 2005, p. 218.

51 John M. Najemy, Machiavelli Between East and West, Diogo Ramada Curto, Eric R. Dursteler, Julius Kirshner et Francesca Trivellato (éd.), From Florence to the Mediterranean and Beyond. Essays in Honour of Anthony Molho, Florence, Olschki, 2009, p. 127-145.

52 Étudié, entre autres, par Tzvetan Todorov, La conquête de l'Amérique. Le problème de l'autre, Paris, Seuil, 1982.

53 Voir aussi Giuseppe Marcocci, « Machiavelli, la religione dei romani e l'impero portoghese ", in Storica, $\mathrm{n}^{\circ}$ 41-42, 2008, p. 35-68. 
"Le seul véritable voyage, le seul bain de Jouvence, ce ne serait pas d'aller vers de nouveaux paysages, mais d'avoir d'autres yeux, de voir l'univers avec les yeux d'un autre, de cent autres, de voir les cent univers que chacun d'eux voit, que chacun d'eux est $"^{54}$.

Lucio Biasiori

Post-doc, Fondation Prix Balzan

Scuola Normale Superiore, Pisa

lucio.biasiori@gmail.com

Je tiens à remercier la Fondation Prix Balzan qui a financé mes recherches, dont ce travail fait partie, et mon camarade et ami Ignazio Veca, qui a traduit ce texte et l'a beaucoup amélioré avec ses critiques.

\section{Résumé}

Machiavel a été toujours considéré comme un classique de la pensée politique européenne. Cet article, tout en abordant la question humaniste de l'imitation du monde ancien, contribue à étudier l'émergence d'une discussion relative au nouveau monde à travers une première comparaison polémique entre l'Europe et les terres nouvellement découvertes. La question qui s'impose est de savoir si cette rencontre s'est limitée à une adaptation du nouveau aux schèmes culturels européens et en quelle mesure la réflexion sur Machiavel a été transformée par le contact avec le nouveau monde.

\section{Mots-clés}

Machiavel, comparaison, découvertes, humanisme.

\section{Abstract \\ Machiavelli has always been treated as a classic of the European political thought. However, his work, although it was grounded on the imitation of the ancient world, contributed to the emergence of a discussion on the new world itself, helping the birth of a first, polemical comparison between Europe and newly-found lands. Was this encounter limited to the well-known adaptation of the unexpected to the European cultural schemas, or was the reflection on Machiavelli himself transformed by the contact with the new world?}

\section{Keywords}

Machiavelli, comparison, great discoveries, humanism. 